Commun. Fac. Sci. Univ. Ank. Series C

V. 6 , pp 235-255 (1988)

\title{
COMPUTER PROGRAM FOR THE TRANSFORMATION OF VES DATA
}

\author{
A.T. BAŞOKUR
}

Ankara University, Faculty of Science, Geophysical Eng., Ankara

\begin{abstract}
A computer program is presented to perform all required rransformations for the interpretation of VES (vertical electrical sounding) data. The program is in BASIC and can be run on microcomputers. The resistivity transform function is computed from the ap parent resistivity data obtained with four types of electrode array (two-electrode, Wenner, Schlumberger and dipole-dipole). The program also allows transformation from one sounding curve obtained any of the above mentioned arrays to others.
\end{abstract}

The program is mainly based on the least-squares approximation of unequally spaced data. Moreover, the forward modelling option which uses a modified version cf the linear filter technique for the unequally spaced data is included.

\section{INTRODUCTION}

In recent years, tremendous developments have been made in computer technology. Among others, with the advance of personal compu ters, in-field processing and interpretation of geophysical data have become possible. Currently, numbers of personal computers employed by geophysical contractors have been progressively increased. Correspondingly, more software will have to be developed by the user since the initial cost of the software offered by relevant companies seems to be expensive for small geophysical contractors, at least in developing countries. In-house development of software is time-consuming and the exact cost is hidden. The only way to reduce the expense is to distribute and exchange software stored on a floppy disk in machine code or to publish source programs.

The purpose of this paper is to present a computer program which performs the fundamental transformations required in the interpretation 
of vertical electrical sounding (VES) data. Namely, the presented program calculates the resistivity transform function from the VES data or an apparent resistivity model curve for any of the two-electrode, Wenner, Schomberger, dipole-dipole arrays. The coefficients obtained from the results of above mentioned transformations permit simultaneous calculation of the corresponding VES data in other three electrode configurations. The program stores the output data on a floppy disk. These stored outputs can be used by other interpreting and plotting programs.

\section{Description of the problem}

There are three types of fundamental transformation problems in the resistivity sounding method. The first one, the so-called forward modelling, is to convert the resistivity transform function to an apparent resistivity curve for a given electrode array to serve the computation of standard model curves. In order to check the validity of the interpretation, this transformation is also required to compare a field curve with a model curve computed using the parameters derived from the result of interpretation. The comparison of field data with a model curve, the so-called iterative interpretation method, can be made by the computer. The comparison is repeated by changing the layer parameters until a satisfactory agreement between the two sets of data is reached.

The second type of transformation is to convert the apparent resistivity data to Koefoed's (1970) resistivity transform function. This function is related with layer parameters through an algebric relation and is independent of the type of array used for the measurements. The interpretation techniques deriving layer parameters from the resistivity transform data is the so-called "direct interpretation". The first step of direct interpretation is to carry out the transformation which is here named as the second type.

The direct interpretation method is based on the reduction of the resistivity transform curve to a lower boundary plane after the calculation of the top layer parameters (thickness and resistivity) at each step. Some proposed direct interpretation techniques are Pekeris (1940), Koefoed (1970), Szaraniec (1980) and Başokur (1984a).

The third type is the transformation of apparent resistivity data from one electrode configuration into another. The transformation of a apparent resistivity data obtained in one electrode configuration to 
Schlumberger data is more important than others, because the avaible computer programs of the iterative interpretation have been mostly prepared for the Schlumberger array.

All above mentioned transformations are performed traditionally by the use of the linear filter method since the pioneering work of Ghosh (1971). The possibility of the application of the convolution theorem for computing resistivity sounding curves has been demonstrated by $\mathrm{Ku}$ netz (1966). Many papers tackling the same problem have been published to perfect the method and filter coefficients. Mostly, they have been summarized by Koefoed (1979).

Recently, Santini and Zambrano (1981) developed a numerical method of calculating the resistivity transform data from Schlumberger apparent resistivity data. Their method is based on the approximation of VES data by a linear combination of suitable fitting functions. In the following years, it has been extended to Wenner and an arbitrary electrode configurations by Kumar and Chowdary (1982), Kohlbeck (1985), respectively. Furthermore, the method is also used for the computation of apparent resistivity model curves for Schlumberger, Wenner and two-electrode arrays (Santini and Zambrano 1983). In all papers related to the method, it has been concluded that the method has advantages over the linear filter method since the equally spaced data is no longer necessary. As a result, approximately equally spaced field data can be input to the computer without interpolation. However, in my opinion, the main advantage of the method is that it allows the computation of three types of transformations in a very effective way by using one single computer program while the linear filter tecnique requires separate computations of each transformation for each electrode array.

The presented computer program uses a generalized version of Santini and Zambrano's (1981) method based on the least-squares approximation of VES data. The linear filter technique has been also used to generate VES model curves to test the computer program and to compare the results of the two methods. The computational scheme of the linear filter method has been modified in order to obtain unequally spaced data. This modification increases the computation time, considerably. However, the linear filter technique is omitted in the routine use of the program.

The program presented here has been designed to perform the three types of transformations for the two-electrode, Wenner, Schlumberger 
and dipole-dipole electrode arrays. But, it may be easily modified to other electrode arrangements. Figure 1 shows the above mentioned electrode arrays and their electrode spacings used traditionally as abscissa in the presentation of the apparent resistivities.

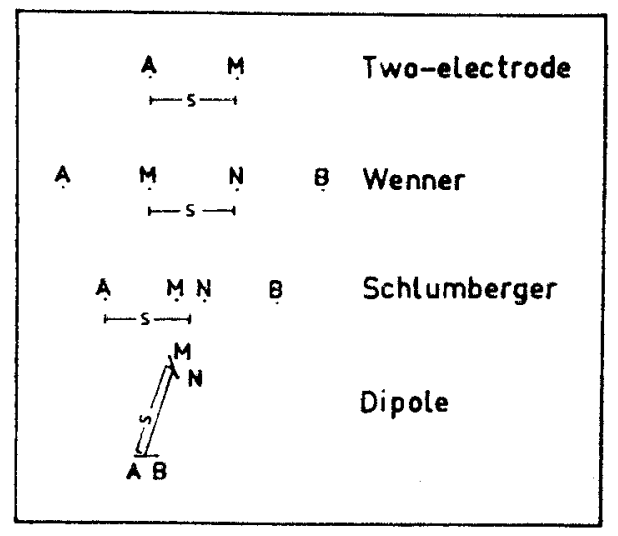

Figure 1. Conventional electrode arrays and corresponding electrode spacings used for the data presentation.

\section{Computations of resistivity sounding curves by the use of linear filter method.}

The first step of all computational methods of apparent resistivity model curves is the same, e.i., the computation of the sample values of the resistivity transform function from a given layer parameters depends on the Pekeris recurrence relation (Pekeris 1940, Koefoed 1979). The second step is the computation of sample values of apparent resistivity from the sample values of the resistivity transform function.

For this purpose, different computational techniques have been offered. In the linear filter technique, the convolution of the resistivity transform function with the sinc response (filter coefficients) which differs according to the used electrode array yields the sample values of apparent resistivity. This argument can be expressed as

$$
\rho_{a}(x)=\mathbf{T}(\mathbf{x}) *[\mathbf{F}(\mathbf{x}) * \mathbf{P}(\mathrm{x})],
$$

where $\rho_{a}(x)$ denotes the apparent resistivity for a given array, $x$ denotes the natural logarithm of electrode spacings shown in Fig. $1, x=1 n$ 
$(1 / \lambda)$ for the resistivity transform function $T(\lambda) . F(x)$ is the filter function and $P(x)$ is the interpolating function (Başokur 1984 b).

The accuracy of the computation depends on the performance of the selected filter. On the other hand, it is difficult to test several published sets of filter coefficients. For this reason, the choice of a particular set depends on personal experience and available computing facilities. In the presented computer program, the filter coefficients of $0^{\prime} \mathrm{Neill}$ and Merrick (1984) have been used for computing two-electrode and Wenner apparent resistivities and the filters of Murakami et al. (1984) and Koefoed (1979) have been used to compute Schlumberger and dipole-dipole apparent resistivities, respectively.

\section{Transformation of VES data using the least-squares technique}

The first step of Santini and Zambroano's (1981) method consists of the approximation of Schlumberger apparent resistivity data by a linear combination of simple fitting functions. The fitting operation is carried out by the least-squares method. Secondly, they construct the Stefanescu kernel function by the linear combination of corresponding fitting functions using the decomposition coefficients of the previous combination.

For the sake of brevity, only the basic theory of the method will be summarized in the following. The reader should refer to the mentioned papers in the manuscript for more details. Here, my main purpose is to show that the determination of the decomposition coefficients for any given array permits the construction of corresponding apparent reșistivities in other electrode configurations and the kernel function as long as analytical relations between input and output fitting functions are derived.

Let us to approximate the Stefanescu kernel by a linear combination of suitable fitting functions; $\mathrm{g}(\lambda ; \varepsilon)$. According to Santini and Zambrano (1982), simple exponential function is the most efficent in fitting the kernel curve:

$$
K^{*}(\lambda)=\sum_{i=1}^{m} \mathbf{b}_{\mathrm{i}} \mathrm{g}\left(\lambda ; \boldsymbol{s}_{\mathrm{i}}\right)
$$

where

$$
\mathrm{g}\left(\lambda ; \varepsilon_{\mathbf{i}}\right)=\exp \left(-\varepsilon_{\mathbf{i}} \lambda\right)
$$

and $K^{*}(\lambda)$ is an approximation of $K(\lambda)$. By defining a new function: 


$$
y(L)=\frac{p_{\mathrm{aL}}(\mathrm{L})-\rho_{1}}{2 \rho_{1}}
$$

and substituting the kernel function in the two-electrode apparent resistivity expression and finally applying inversion formulas of Hankel transforms, we get

$$
\mathbf{y}^{*}(L)=\sum_{i=1}^{m} b_{i} \mathbf{f}\left(L ; \varepsilon_{i}\right)
$$

where

$$
\mathbf{f}\left(\mathrm{L} ; \varepsilon_{\mathrm{i}}\right)=\mathrm{L} /\left(\varepsilon_{\mathrm{i}}^{2}+\mathbf{L}^{2}\right)^{1 / 2}
$$

The appropriate fitting functions for Wenner, Schlumberger and dipole-dipole arrays may be derived by using existing relationships between apparent resistivity functions of two electrode and one of the other arrays while keeping $b_{i}$ and $\varepsilon_{i}$ coefficients unchanged. The results are

$$
\begin{aligned}
& \mathbf{f}\left(\mathbf{a} ; \varepsilon_{i}\right)=2 \mathbf{a} /\left(\varepsilon^{2}+\mathbf{a}^{2}\right)^{1 / 2}-2 \mathbf{a} /\left(\varepsilon^{2} \mathbf{i}+4 \mathbf{a}^{2}\right)^{1 / 2}, \\
& f\left(s ; \varepsilon_{i}\right)=s^{3} /\left(\varepsilon^{2} i+s^{2}\right)^{3 / 2}, \\
& \mathbf{f}\left(\mathbf{R} ; \varepsilon_{\mathrm{i}}\right)=\mathbf{R}^{3}\left(\mathbf{R}^{2}+(1-3 \mathbf{p}) \varepsilon^{2} \mathbf{i}\right) /\left(\varepsilon_{\mathrm{i}}^{2}+\mathbf{R}^{2}\right)^{5 / 2} \\
& =\left\{1+(1-3 \mathbf{p})\left(\varepsilon_{\mathbf{i}} / \mathbf{R}\right)^{2}\right\} /\left(1+\left(\varepsilon_{\mathbf{i}} / \mathbf{R}\right)^{2}\right)^{5 / 2},
\end{aligned}
$$

where $p$ is a constant defining the type of dipole-dipole configuration. Except the dipole--dipole fitting function, the other ones have heen published (Santini and Zambrano 1981, 1983).

The observed VES data should be extrapolated both towards the left and the right up to almost reaching the asymtotes $p_{1}$ and $p_{n}$, respectively. The first and last values of $\varepsilon_{i}$ are taken approximately equal to the first abscissae of the apparent resistivity and half of the last abscissae value of the apparent resistivity, respectively. Thus, the number of fitting functions $(m)$ and $\varepsilon_{i}$ values can be calculated by distributing equally spaced five fitting functions over one logarithmic cycle.

The calculation of the number of fitting functions and $\varepsilon_{i}$ values is more complex for the computation of the apparent resistivity model curves. The procedure to compute $m$ and $\varepsilon_{i}$ is well described by Santini and Zambrano $(1981,1983)$. However, all these computations are carried out automatically by the presented program. 
It is obvious from the above equations, if the sample values of a VES field or model curve is known then the corresponding VES data in other electrode arrays and the Stafanescu kernel function (consequently the resistivity transform) can easily be obtained using the same decomposition coefficients.

\section{Case of perfectly insulating and conducting substratum}

In the case of a perfectly insulating substratum, the fitting function given above could not show the same behaviour with the apparent resistivity curves and the kernel function, because the VES data will approach an asymptote with a slope of $45^{\circ}$ at large abscissa values. The set of fitting functions which shows the same property with VES data may be given as:

$$
\begin{aligned}
& \mathrm{g}\left(\lambda ; \varepsilon_{\mathrm{i}}\right)=\exp \left(-\varepsilon_{\mathrm{i}} \lambda\right) / \varepsilon_{\mathrm{i}} \lambda, \\
& \mathbf{f}\left(\mathbf{a} ; \varepsilon_{\mathbf{i}}\right)=\left(2 \mathbf{a} / \varepsilon_{\mathbf{i}}\right)\left\{\operatorname{Ln}\left[\varepsilon_{\mathbf{i}}+\left(4 \mathbf{a}^{2}+\varepsilon_{\mathbf{i}^{2}}\right)^{1 / 2}\right]\right. \\
& \left.-\operatorname{Ln}\left[\varepsilon_{\mathrm{i}}+\left(\mathbf{a}^{2}+\varepsilon_{\mathrm{i}^{2}}^{2}\right)\right]\right\} \text {, } \\
& f\left(s ; \varepsilon_{i}\right)=\left(s / \varepsilon_{i}\right)\left[1-\varepsilon_{i} /\left(\varepsilon_{i}^{2}+s^{2}\right)^{1 / 2}\right] \text {, } \\
& f\left(R ; \varepsilon_{i}\right)=\left(R / \varepsilon_{i}\right)(1-p)\left[1-\varepsilon_{i} /\left(\varepsilon_{i}^{2}+R^{2}\right)^{1 / 2}\right] \\
& -p R^{3} /\left(\varepsilon_{\mathrm{i}}{ }^{2}+\mathbf{R}^{2}\right)^{3 / 2} \text {. }
\end{aligned}
$$

As can be noted, a fitting function for the two-electrode array is not given since the integral equation for this array does not converge in this case (Santini and Zambrano 1983).

Similarly, for the particular case of conducting substratum, it is necessary to use the fitting functions which tend to zero in the same way as VES data for large abscissa values. These functions may be derived from Santini and Zambrano (1982) as follows:

$$
\begin{aligned}
& g\left(\lambda ; \varepsilon_{i}\right)=1-\exp \left(-\varepsilon_{i} \lambda\right) \\
& \mathbf{f}\left(\mathbf{L} ; \varepsilon_{i}\right)=1-\mathbf{L} /\left(\varepsilon_{i}{ }^{2}+L^{2}\right)^{1 / 2} \\
& \mathbf{f}\left(a ; \varepsilon_{i}\right)=1-\left[2 a /\left(\varepsilon_{i}{ }^{2}+a^{2}\right)^{1 / 2}-2 a /\left(\varepsilon^{2}{ }_{i}+4 a^{2}\right)^{1 / 2}\right] \\
& f\left(s ; \varepsilon_{i}\right)=1-s^{3} /\left(\varepsilon_{i}{ }^{2}+s^{2}\right)^{3 / 2} \\
& f\left(R ; \varepsilon_{i}\right)=1-R^{3}\left(R^{2}+(1-3 c) \varepsilon_{i}{ }^{2}\right) /\left(\varepsilon_{i}{ }^{2}+s^{2}\right)^{5 / 2}
\end{aligned}
$$

Algorithm description and usage

The computer program has been written in Microsoft BASIC (BASICA, version 3.0) and it runs under the DOS operating system and IBM- 
PC or compatible microcomputers. In the program, an algorithm which solves a system of simultaneous linear equations with symmetric coefficient matrix has been adapted and converted to BASIC from a FORTRAN IV subroutine "GELS" (reprinted by permission from system/360 Scientific Subroutine Package (1968) by International Business Machines Corporation).

The statements employed in the computer program are supported both BASIC interpreter and compiler. Execution under interpreter is slow. It is recommended to compile the program and obtain an executable program under the operating system. During the compilation of the program, /E switch should be activated to handle "ON ERROR GOTO" and "RESUME" statments (refer to the Microsoft BASIC compiler user's guide). The minumum memory requirement for the compiler is $64 \mathrm{~K}$.

At the runtime, the program instructs the user and asks only the necessary questions. It also checks inputs and repeats a question until it obtains a reasonable answer. Surely, the sample values of VES data and electrode spacings cannot be checked. The program poses the following questions may be omitted as long as they are unnecessary.

Question 1: "Give data filename or write end". The program asks the caption. It may consist of any number of characters. However, the first eight characters are used as the filename to access the VES data on floppy disk. The first character of the caption shouldn't be numeric. If a file with the same filename already exists on the disk, any existing data in the file will be destroyed and new data will be saved if one answers question 4 positively. If one writes "END" then the program is terminated.

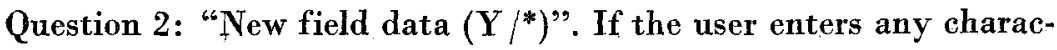
ter except "Y" or " $y$ " then previously stored data on the disk are accessed and are printed on the display. In all cases, the program branches to question 3.

Question 3: "Enter (the key number) spacing and app. res. $>$ ". A pair of numbers, electrode spacing and corresponding apparent resistivity value are entered leaving space between each number. The data sets can be given in any order since the data are sorted according to the electrode spacings before proceeding to the next stage. To correct any data entries, the key number, the correct electrode spacing and apparent resistivity values separated by a space are entered. The way to 
cancel data entries is similar to the correction procedure. Zero is assigned to the electrode spacing or to the apparent resistivity value. (R) option is choosen to re-initialize the program when the user accidentally access an undesired file. (P) option is used to display the sorted data. (C) or (S) options are choosen to proceed to the next stage while (S) option permits to store the data on the disk under the name given in question 1. The extension "DAT" is automatically added to the filename.

Question 4: At this question, the type of electrode array is declared by entering 1 for the resistivity transform function, 2 for the twoelectrode, 3 for Wenner, 4 for Schlumberger and 5 for the dipole-dipole apparent resistivities. If the input is the resistivity transform function the program computes apparent resistivity model curves for electrode arrays which will be declared in question 5 .

Question 5: "Give the desired outputs Yes $=0$ ". This question enables the user to select outputs. Selection is made by pressing the "RETURN" key when the name of any electrode array has appeared. If the user enters a number other than zero, the computations for this array are omitted. Finally, the last question of this session "Correct $\left(\mathrm{Y} /{ }^{*}\right)^{\text {" }}$ allows to correct errors which have been made in questions 4 and 5 .

Question 6: At this question, the type of VES data is entered. The options are perfectly conductive substratum $(-1)$, the substratum with finite resistivity $(0)$ and perfectly resistive substratum (1). The resistivity of the top layer is also asked.

Question 7: "Continue $\left(\mathrm{Y} / /^{*}\right)$ ". After the above questions have been answered, the program will begin to approximate the input data by fitting functions. The results will be listed on the display together with this question. If the difference between field data and approximated data is very high, the results are not acceptable, and then the user may answer this question negatively. In that case, the program will return to the question 1 and all calculated data will be destroyed. Otherwise, the user enters "Y" and the program produces a hard copy via the line printer.

Question 8: "Store the outputs on floppy disk $(Y / * ")$. This question allows the storing of outputs on the disk. The filenames of outputs are adjusted by keeping the first seven characters of the filename of the input data and by adding one character to the end of it. These added characters are " $R$ " for the resistivity transform function, " $T$ " for two- 
electrode array, "W" for Wenner array, "S" for Schlumberger array and "D" for the dipole-dipole array.

After all these questions have been answered, the program computes all desired outputs and produces hard copies via the line printer.

\section{Test mode}

In the above, normal usage of the program has been described to transform the VES field data obtained in one electrode configuration to another configuration and to calculate the resistivity transform curve from VES data or to compute apparent resistivity model curves by means of the least-squares technique. To test the performance of the program and accuracy of the method, the test mode is provided. In the test mode, a model curve is generated using the linear filter method and is fed to the computer as an input. For operating the program in the test mode, the first sample value of apparent resistivity is assigned to minus one. Then, the program asks the layer parameters between questions 5 and 6. The sample values of the model curve and the VES data computed by the least - squares method as well as relative errors between data groups will be printed. A small relative error indicates the good performance of the method.

If the input is the resistivity transform function, the calculation of VES model curves in the test mode is made by the use of both the linear filter and least-squares methods. In this case, relative errors on the printout indicates the relative difference between the two methods.

The program can also be used to obtain the printout of a VES model curve. The type of electrode array is selected in question 4 and the first sample value of apparent resistivity data is assigned to minus one. Question 5 is answered negatively except the electrode array selected in question. 4. Then, the program computes the sample values of a model VES curve by the use of the linear filter technique.

\section{Example}

The results of a demonstration run will be presented so that the user can check and edit the program until correct results are obtained.

The first and second columns of Table 1 present electrode spacings and coresponding apparent resistivity values for Schlumberger array for the case where the layer resistivities are $1,20,0.1,1 \mathrm{ohm}-\mathrm{m}$ and 
Table 1. Schlumberger apparent resistivity data (APR. RES.), approximated data (COM. APR. RES.) and the relative errors between the two sets of data.

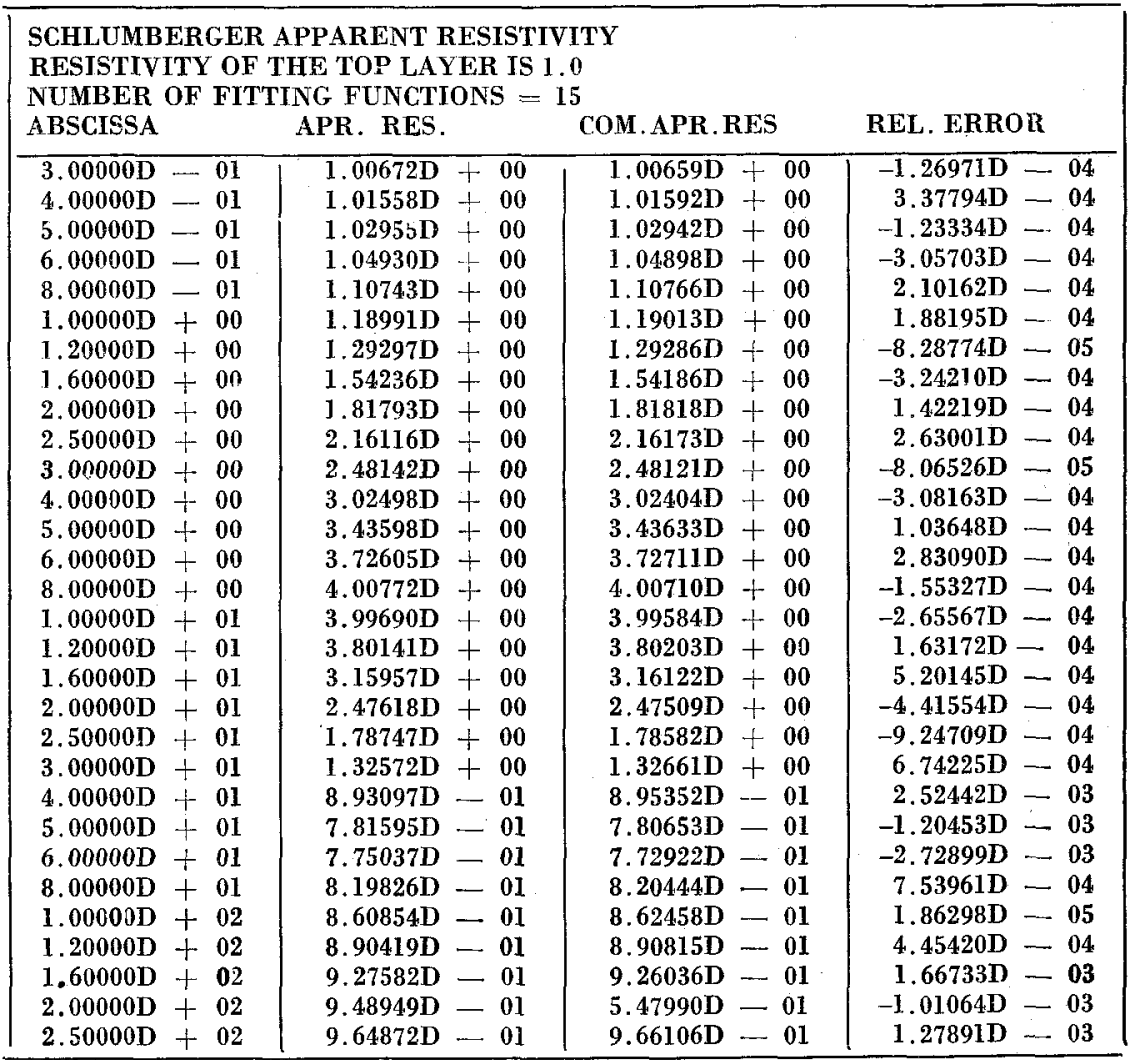

thicknesses are 1,2, 3 meters. The computation of Schlumberger apparent resistivity was carried out by the linear filter method and the results were stored on disk by using the presented program in the mode described on the last paragraph of the above section. At the second run of the program, these values were fed to the computer as the input simulating a field curve. The approximated apparent resistivity (COM. APR. RES) shown on the third column of Table 1 and relative errors shown on the fourth column were obtained. As the relative errors were small, question was answered affirmatively to let the computer continue the calculation of the outputs. The corresponding outputs, namely the sample values of the resistivity transform function and the apparent resistivities, are presented in Table 2. In daily usage, the VES data of each electrode array are printed on separate pages. 
Table 2. Corresponding apparent resistivities for the resistivity transform frunction $T^{*}(1 / \lambda)$, the two electrode apparent resistivity $\rho_{a}{ }^{*}(\mathrm{~L})$, the Wenner apparent resistivitv $p_{a}{ }^{*}(a)$ and the radial dipole apparent resistivity $\rho_{a}{ }^{*}(\mathbf{R})$.

\begin{tabular}{|c|c|c|c|c|}
\hline ABSCISSA & $\mathrm{T}^{*}(1 / \lambda)$ & $P_{a}^{*}(\mathrm{~L})$ & $P_{a}{ }^{*}(a)$ & $P_{a}{ }^{*}(\mathrm{R})$ \\
\hline $3.00000 \mathrm{D}-01$ & $1.00183 \mathrm{D}+00$ & $1.34969 \mathrm{D}+00$ & $1.01942 \mathrm{D}+00$ & $9.94566 \mathrm{D}-01$ \\
\hline $4 . c 0000 \mathrm{D}-01$ & $1.01178 \mathrm{D}+00$ & $1.42673 \mathrm{D}+00$ & $1.043310+00$ & $9.93915 \mathrm{D}-01$ \\
\hline $5.00000 \mathrm{D}-01$ & $1.03323 \mathrm{D}+00$ & $1.57298 \mathrm{D}+00$ & $1.07928 D+00$ & $9.88580 \mathrm{D}-01$ \\
\hline $6.00000 \mathrm{D}-01$ & $1.066410+00$ & $1.67997 \mathrm{D}+00$ & $1.12727 \mathrm{D}+00$ & $9.80414 \mathrm{D}-01$ \\
\hline $8.00000 \mathrm{D}-01$ & $1.16015 \mathrm{D}+00$ & $1.88215 \mathrm{D}+00$ & $1.25465 \mathrm{D}+00$ & $9.65370 \mathrm{D}-01$ \\
\hline $1.00000 \mathrm{D}+00$ & $1.27763 \mathrm{D}+00$ & $2.06669 \mathrm{D}+00$ & $1.41352 \mathrm{D}+00$ & $9.57087 \mathrm{D}-01$ \\
\hline $1.20000 \mathrm{D}+00$ & $1.40822 D+00$ & $2.23266 \mathrm{D}+00$ & $1.59123 \mathrm{D}+00$ & $9.58220 \mathrm{D} \_01$ \\
\hline $1.60000 \mathrm{D} \div 00$ & $1.68327 \mathrm{D}+00$ & $2.50965 \mathrm{D}+00$ & $1.96370 \mathrm{D}+00$ & J.00500D+00 \\
\hline $2.00000 \mathrm{D}+00$ & $1.95282 \mathrm{D}+00$ & $2.73985 \mathrm{D}+00$ & $2.32107 \mathrm{D}+00$ & $1.11911 \mathrm{D}+00$ \\
\hline $2.50000 \mathrm{D}+00$ & $2.26066 \mathrm{D}+00$ & $2.90518 \mathrm{D}+00$ & $2.71844 \mathrm{D}+00$ & $1.23750 \mathrm{D}+00$ \\
\hline $3.00000 \mathrm{D}+00$ & $2.52572 \mathrm{D}+00$ & $3.02337 \mathrm{D}+00$ & $3.052 .92 \mathrm{D}+00$ & $1.56854 \mathrm{D}+00$ \\
\hline $4.00000 \mathrm{D}+00$ & $2.91706 \mathrm{D}+00$ & $3.11863 \mathrm{D}+00$ & $3.5399 \mathrm{JD}+00$ & $2.07051 D+01$ \\
\hline $5.00000 \mathrm{D}+00$ & $3.14042 D+00$ & $3.09193 \mathrm{D}+00$ & $3.81743 \mathrm{D}+00$ & $2.56288 \mathrm{D}+00$ \\
\hline $6.00000 \mathrm{D}+00$ & $3.23606 \mathrm{D}+00$ & $2.99383 D+00$ & $3.931210+00$ & $3.02279 \mathrm{D}+03$ \\
\hline $8.00000 \mathrm{D}+00$ & $3.20008 \mathrm{D}+00$ & $2.69736 \mathrm{D}+00$ & $3.82882 \mathrm{D}+00$ & $3.78008 \mathrm{D}+00$ \\
\hline $1.00000 \mathrm{D}+01$ & $3.03064 \mathrm{D}+00$ & $2.36643 \mathrm{D}+00$ & $3.48502 \mathrm{D}+00$ & 4. $29075 \mathrm{D}+00$ \\
\hline $1.20000 \mathrm{D}+01$ & $2.82974 \mathrm{D}+00$ & $2.05644 \mathrm{D}+00$ & $3.054 .47 \mathrm{D}+00$ & $4.57175 \mathrm{D}+00$ \\
\hline $1.60000 \mathrm{D}+01$ & $2.46407 \mathrm{D}+00$ & $1.56590 \mathrm{D}+00$ & $2.23425 \mathrm{D}+00$ & $4.5724 .4 \mathrm{D}+00$ \\
\hline $200000 \mathrm{D}+01$ & $2.18485 \mathrm{D} \div 00$ & $1.24783 \mathrm{D}+00$ & $1.63222 \mathrm{D}+00$ & $4.07898 \mathrm{D}+00$ \\
\hline $2.50000 \mathrm{D}+01$ & $1.93533 \mathrm{D}+00$ & $1.02563 \mathrm{D}+00$ & $1.17883 \mathrm{D}+00$ & $3.21176 \mathrm{D}+00$ \\
\hline $3.000000+01$ & $1.76032 \mathrm{D}+00$ & $9.20278 \mathrm{D}-01$ & $9.48150 \mathrm{D} \ldots 01$ & $2.39865 \mathrm{D}+0 \mathrm{r}$ \\
\hline $4.00000 \mathrm{D}+01$ & $1.53815 \mathrm{D}+00$ & $8.63433 \mathrm{D}-01$ & $8.01280 \mathrm{D}-0 \mathrm{l}$ & $1.33772 \mathrm{D}+0 \mathrm{r}$ \\
\hline $5.00000 \mathrm{D}+.0 \mathrm{l}$ & $1.40693 \mathrm{D}+00$ & $8.72429 \mathrm{D} \longrightarrow 0 \mathrm{I}$ & $7.98177 \mathrm{D}-01$ & $8.82984 \mathrm{D}-01$ \\
\hline $6.00000 \mathrm{D}+01$ & $1.32225 \mathrm{D}+00$ & $8.92405 \mathrm{D}-01$ & $8.24130 \mathrm{D}-01$ & $7.31420 \mathrm{D}-01$ \\
\hline $8.00000 \mathrm{D}+01$ & $1.22206 \mathrm{D}+00$ & $9.25586 \mathrm{D}-01$ & $8.73052 \mathrm{D}-01$ & $7.20922 \mathrm{D}-01$ \\
\hline $1.00000 \mathrm{D}+02$ & $1.16650 \mathrm{D}+00$ & $9.46681 \mathrm{D}-01$ & $9.04947 \mathrm{D}-01$ & 7,76728D—01 \\
\hline $1.20000 \mathrm{D}+02$ & $1.13210 \mathrm{D}+00$ & $9.60681 \mathrm{D}-01$ & $9.26368 \mathrm{D}-01$ & $8.20564 \mathrm{D}-01$ \\
\hline $1.60000 \mathrm{D}+02$ & $1.09289 \mathrm{D}+00$ & $9.78120 \mathrm{D}-01$ & $9.53735 \mathrm{D}-01$ & $8.72303 \mathrm{D}-01$ \\
\hline $2.000000+02$ & $1.07185 D+00$ & $9.88414 \mathrm{D} \longrightarrow 01$ & $9.70436 \mathrm{D}-01$ & $9.03132 \mathrm{D}-01$ \\
\hline $2.50000 \mathrm{D}+02$ & $1.05661 \mathrm{D}+00$ & $9.96262 \mathrm{D}-01$ & $9.83478 \mathrm{D}-01$ & $9.29811 \mathrm{D}-01$ \\
\hline
\end{tabular}

\section{Remarks}

It should be kept in mind that the use of the least-squares method requires more knowledge about the nature of the method than the linear filter method. Accuracy depends on the abscissa range and type of selected fitting functions. On the contrary, to use a computer program based on the linear filter technique, the user should only know the extrapolation of the VES curve is required to the left as well as to the right. For this reason, I recommend the user to refer to previously mentioned papers for a detailed understanding of the method.

Moreover, the program may produce incorrect results in the computation of Wenner apparent resistivity by the linear filter method at large abscissa values for the case where the substratum is perfectly conductive or resistive. A set of Wenner filter coefficients which gives very accurate 


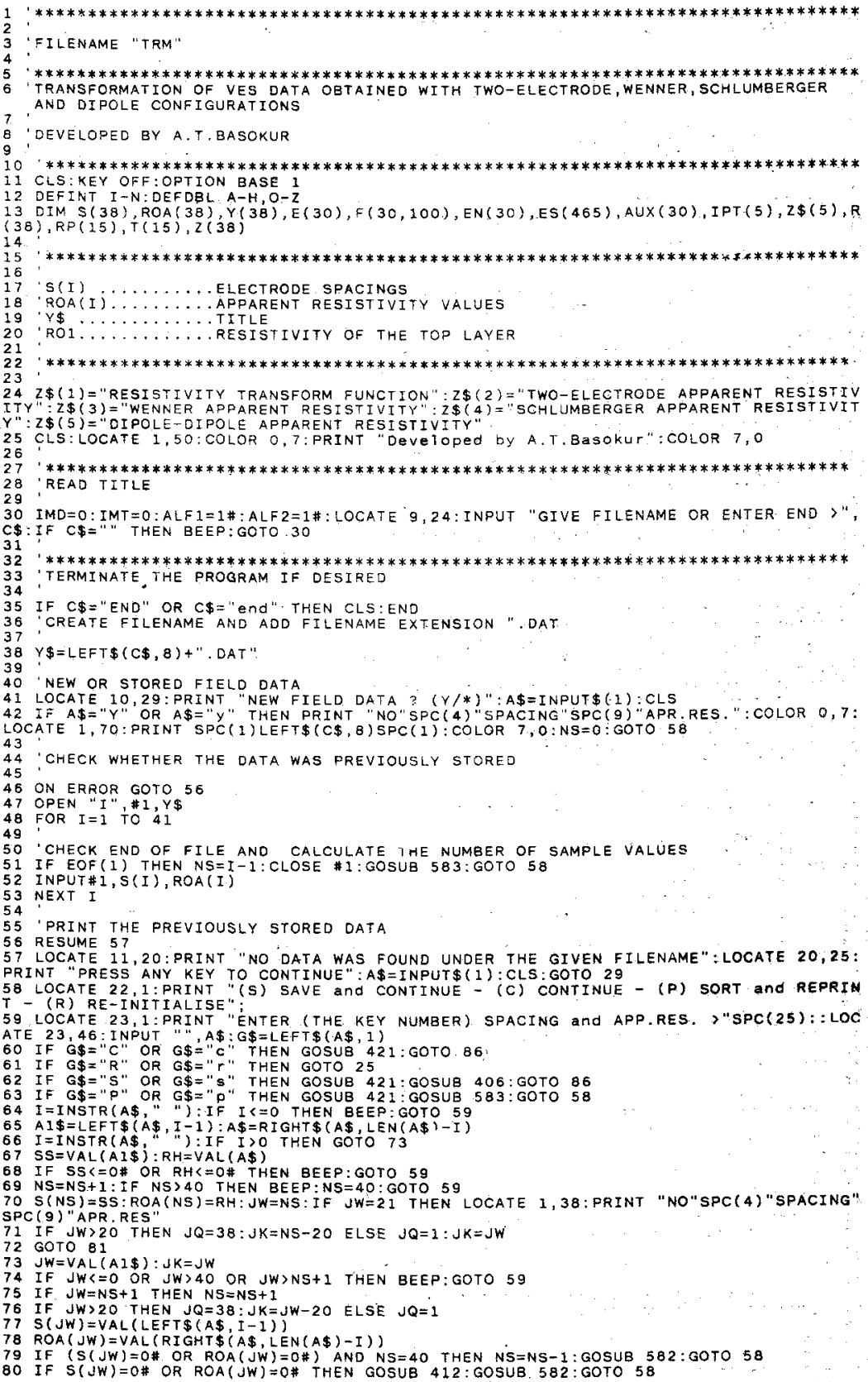


81 LOCATE JK+1, JQ:PRINT SPC $(35)$ : LOCATE JK+1, JQ:PPINT USING "\#"; JW; : PRTNT TAE (JQ +4)" "; PRINT USING "\#\#\#. \#"; S(JW);:PRINT TAB (JQ+20)" ";:PRINT USING" "\#\#\#\#\#\#": 82 COTO 


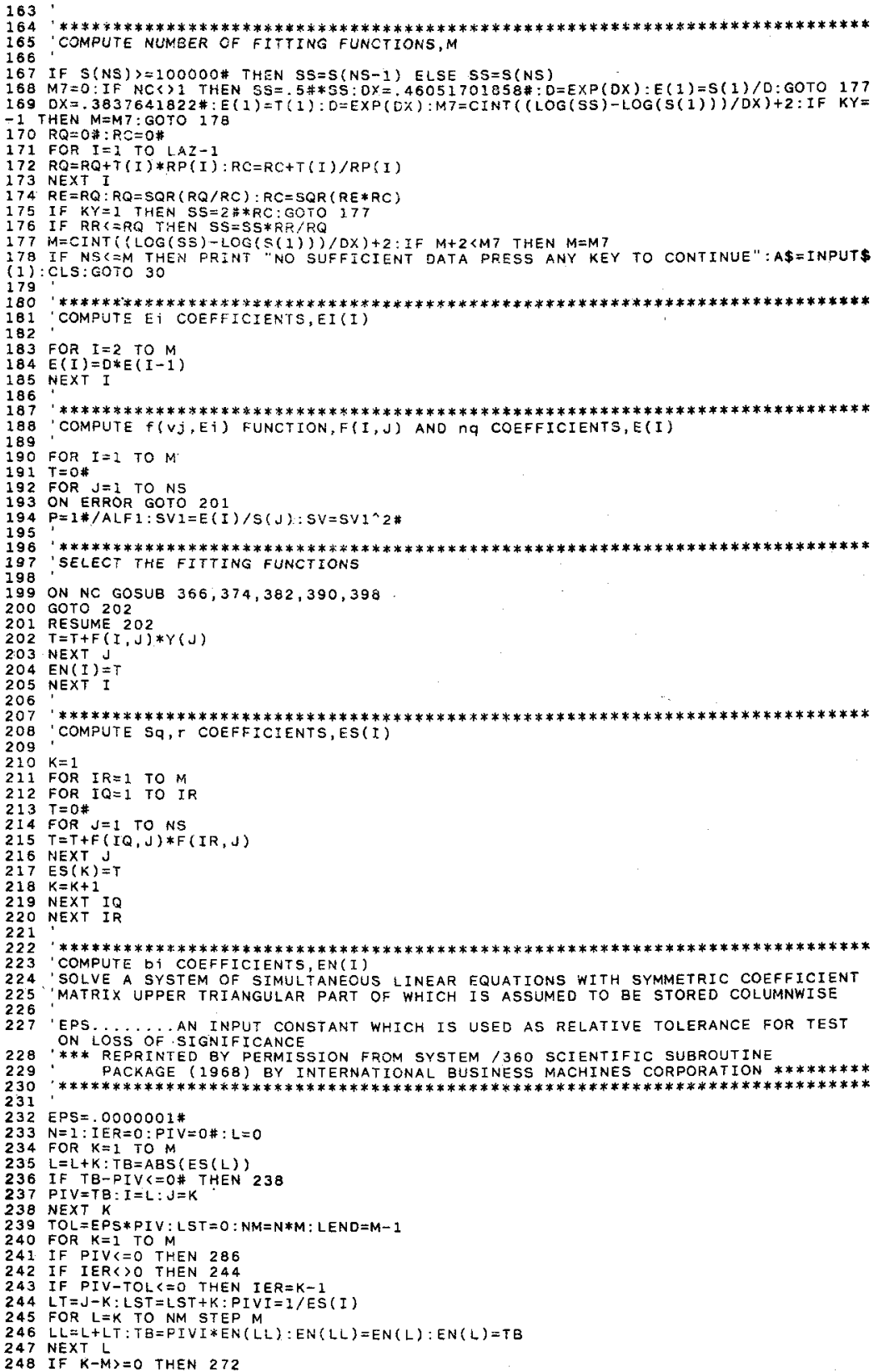




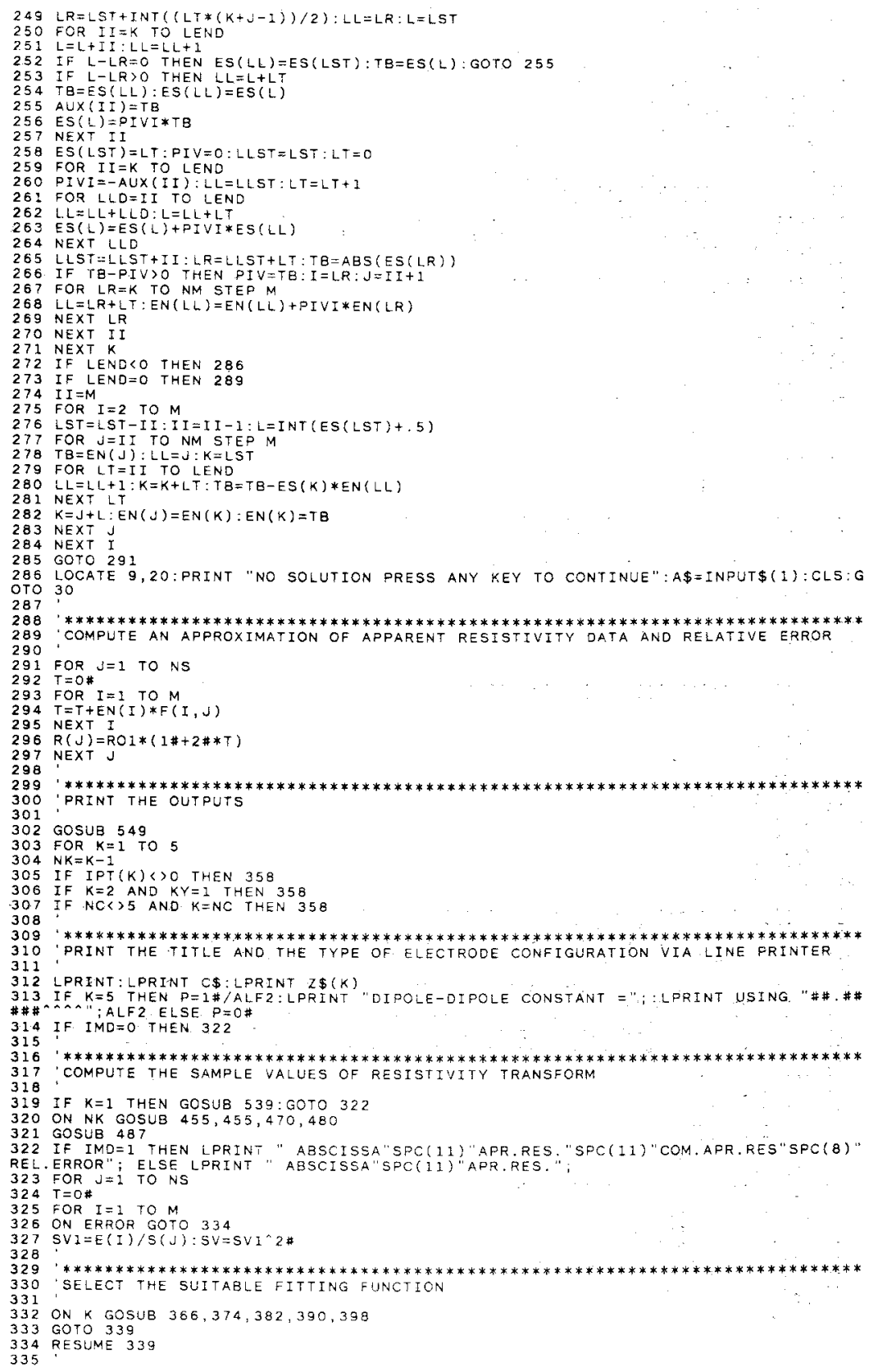




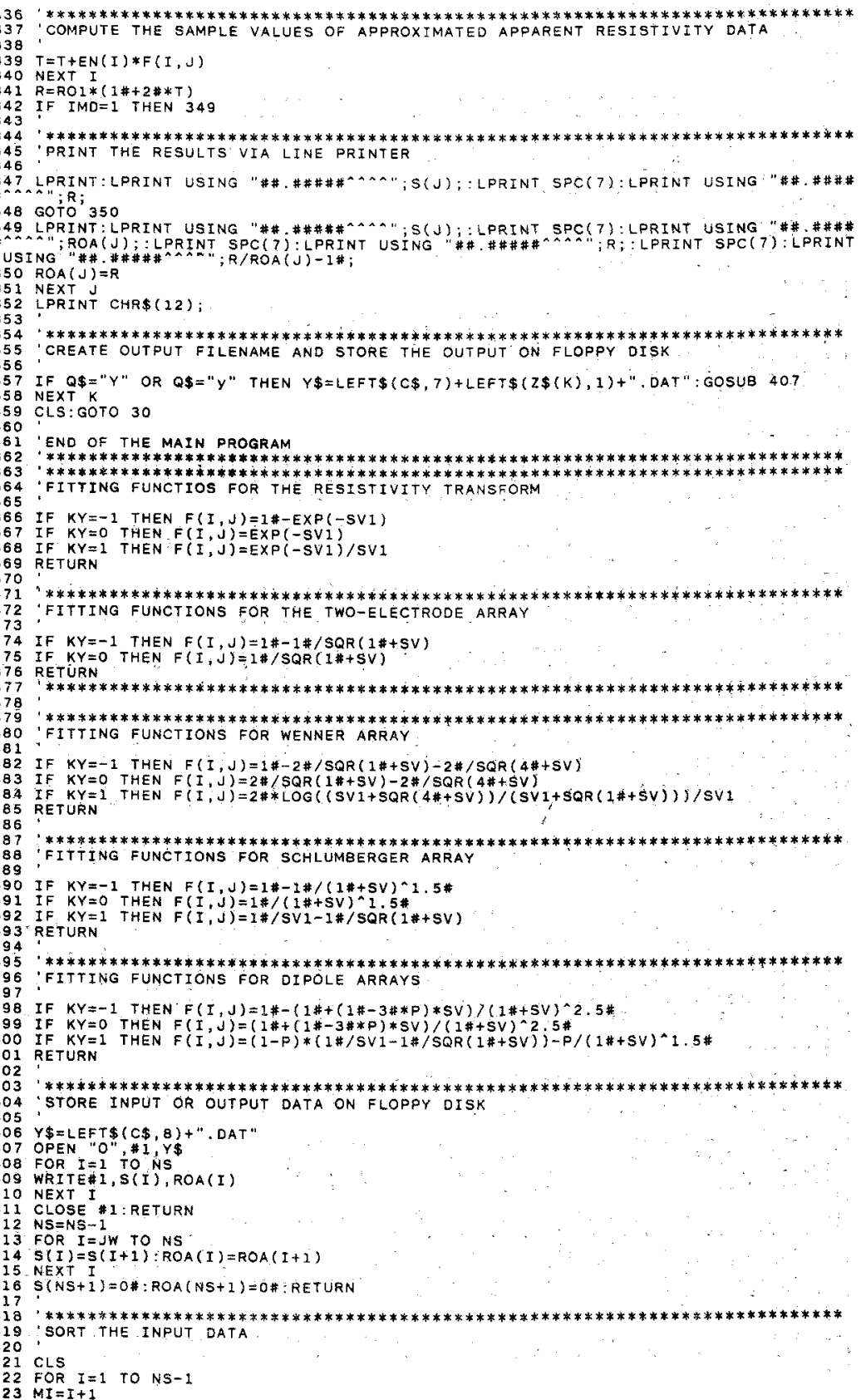


424 FOR IJ=MI TO NS

425 IF $S$ (I) $<S$ (IJ) THEN 427

426 SWAP S(I), S(IJ):SWAP ROA(I), ROA(IJ)

427 NEXT IJ

428 NEXT I

429 RETURN

430

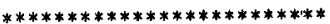

432 READ LAYER PARAMETERS, RESISTIVITY AND THICKNESS OR DEPTH.

434 LOCATE 2,3:PRINT "CHOICE THICKNCSS (T) OF DEPTH (D)":BI\$=INPUT\$(1):IF B1\$="T OR BI\$="D" THEN 435 ELSE BEEP:GOTO 434

435 LOCATE 43 : PRINT " GIVE NUMBER OF THE LAYER $\gamma " \operatorname{SPC}(3):$ LOCATE $4,39:$ IN

PUT ". LAZ: IF LAZ<2 THEN GOTO 434

436 PRINT

437 FOR I=1 TO LAZ - I

439 PRINT " GIVE RESISTIVITY AND THICKNESS/DEPTH OF THE " ;:PRINT USING "\#\#"; I;:I NPUT "TH LAYER > "RF(I), Y(I)

4 40 NEXT I

441 PRINT " GIVE THE RESISTIVITY OF IAST LAYER " , RR

443 LOCATE 22,35:PFINT "CORRECT (Y/*)" A $\$$ =TNPUT\$(I) 434

445 IF LAZ<3 THEN RETURN

446 IF B1\$="T" THEN RETURN

447 FOR $I=L A Z-1$ TO 2 STEP - 1

$448 T(I)=T(I)-T(I-1)$

449 NEXT I

450 RETURN

451

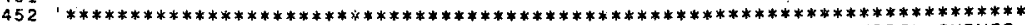

453 ' $F I L T E R S$ TO COMPUTE THE SAMPLE VALUES OF APPARENT RESISTIVITY MODEL CURVES FOR TWO-ELECTRODE, WENNER, SCHLUMBERGER ANO DIPOLE ARRAYS

IQ (Number of the filter coeficients), II (Number of filtar coefficients which have positive key number). ZM (Number of the sampling intervals per dacade), $x$ (horizontal shift)

456

457 **************************************************************************

Filter coefficients of $O^{\prime}$ NEILL and MERRICK-Geoph. Prosp. $v=30, p=112$ for

computing Two-Electrode or Wenner apparent reststivity model curves

460 IQ $=36:$ IIF $=10: Z M=6 \#: X=-046339794$ : $E E=E X P(2.3025350929 \# / Z M)$

461 (1) : I $-19282817 \#: Z(6)=51481121 \#: Z(7)=-.53249164 * Z(B)=-.32349971 *: Z(9)=.1586253 \#: 2(1$ $0)=.29934872 *$

$462 Z(11)=29147527 * Z(12)=22053809 * Z(13)=.15458954 * Z(14)=.11087382 * Z: 2(15)=.0$ $80094716 *: Z(16)=.05186661 *: Z(17)=037878738 * Z(18)=023921121 *$

$80094716 *: 2(16)=.05186661 \%: Z(17)=.037878738 \%: Z(18)=.023921121 \%$

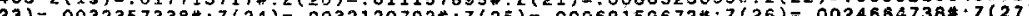
23) $0.0032357338: 2(24)=.00321207924$

$464 Z(29)=-.0016292507 \#: Z(30)=002685288 \#: Z(31)=-0024133139 \#: Z(32)=.0026864314 \#$ $: Z(33)=-.0022372433 \#: Z(34)=.0018339484: Z(35)=-.0010097715 \#: Z(36)=.00039053314 *$ 465 RETURN

466 - $467 * * * * * * * * * * * * * * * * * * * * * * * * * * * * * * * * * * * * * * * * * * * * * * * * * * * * * * * * * * * * * * * * * * * * * * * *$

468 . 'Fitier coefficients of MURAKAMI for computing schiumbergar apparent

469

Filter coefficients of MUR
resistivity modal curves

470 IQ=28:IIF=19:ZM=6*:X=.13072093886*:EE=EXP(2.3025850929\%/ZM)

$471 Z(1)=.000086463368 * Z(2)=-.00036875438 *: Z(3)=.00092111524 *: Z(4)=-.0018772686$ * $Z(5)=.0035343506 *: Z(6)=-.0064612278 *: Z(7)=.011691267 * Z(8)=-.021088677 \%$

$472 Z(9)=.038045092: Z(10)=-068913666: Z(11)=.12655755: Z(12)=-.2435547: 2(13)=$ $.52117305 \%: Z(14)=1.2644217 *: Z(15)=2.7992503 *: Z(16)=-3.4653734$

$473 Z(17)=.41912647 \%: Z(18)=1.1950174 \$ Z Z(19)=.6107326 *: Z(20)=.24298434 *: Z(21)=.08$ $2207576 *: Z(22)=.027770876 *: Z(23)=.0087075202 *: 2(24)=.0028615354 *$ $2207576 * Z Z(22)=.027770876 *: Z(23)=0087075202 *: Z(24)=0020615354 *$
$474 Z(25)=.00088398812 *: 2(26)=.00028020133 *: Z(27)=.00010060054 *: 2(28)=.006034147$
$278 *$

475 RETURN

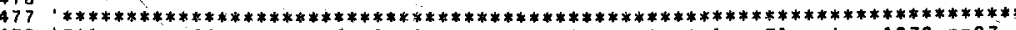

478 'Filter coefficients of KOEFOED Geosounding Principles Elsevier $1979 p=97$

479 for computing Dipole-Difole apparent resistivity model curves

$480 I Q=30: I I F=17: Z M=6 \#: X=.1051 *: E E=E X P(2.3025850929 * / Z M)$

$481 Z(1) *-.001 *-P * .001 *: Z(2)=.0061 *+P * .0082 \#: Z(3)=-.0182 *-P * .0346 \#: Z(4) \approx .0381 *+P$ $* .1018 *: Z(5)=-.0643 *-P * .2404 * Z Z(\delta)=.0935 \$+P * .4915 *: Z(7)=-.1223 *-P * .915 *: Z(B)=.14$ $72 \#+P * 1.6079 *: Z(9)=-.1607 \#-P * 2.7371 *: Z(10)=-1368 *+P * 4.5747 \#: Z(11)=.0298 *-P * 7.351$ $4 \$$

$482 z(12)=-.6949 \#+P * 9,9485 * Z(13)=2,3397 *-P * 6,7406 * Z Z(14)=-3.4031 *-P * 6.8271 * Z(1$ $5)=.5326 \#+P * 15,1423 * Z(16)=1.3371 *-P * 7.172 \#: Z(17)=.2972 *+P * 2.886 \#: Z(18)=.6032 \#-P$ $* 3.2823 * Z(19)=-388 *+P * 1.5391 * Z(20)=5669 \#-P * 1.1701 * Z(21)=-.5859 \#+P *, 5929 *$

$483 z(22)=6107 \#-F^{*}, 2915 \# Z Z(23)=-5724 *+P * 0642 * Z z(24)=4921 *+P * 0563 \#: 2(25)=-.3$ 79*-F* $1093 *: Z(26)=2575 * P * .1096 \# ; z(27)=-149 \#-P * .0909 *: Z(28)=.069 *+P * .0443 \#: Z 6$ $29)=-.0226 *-p * .0164 * 2(30)=.0038 \#+P * .003 \#$

434 RETURN

485

485

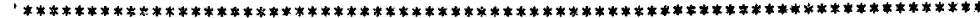
488

COMPUTE TME SAMPLE VALUES OF THE RPPARENT RESISTIVITY MODEL CURVE 


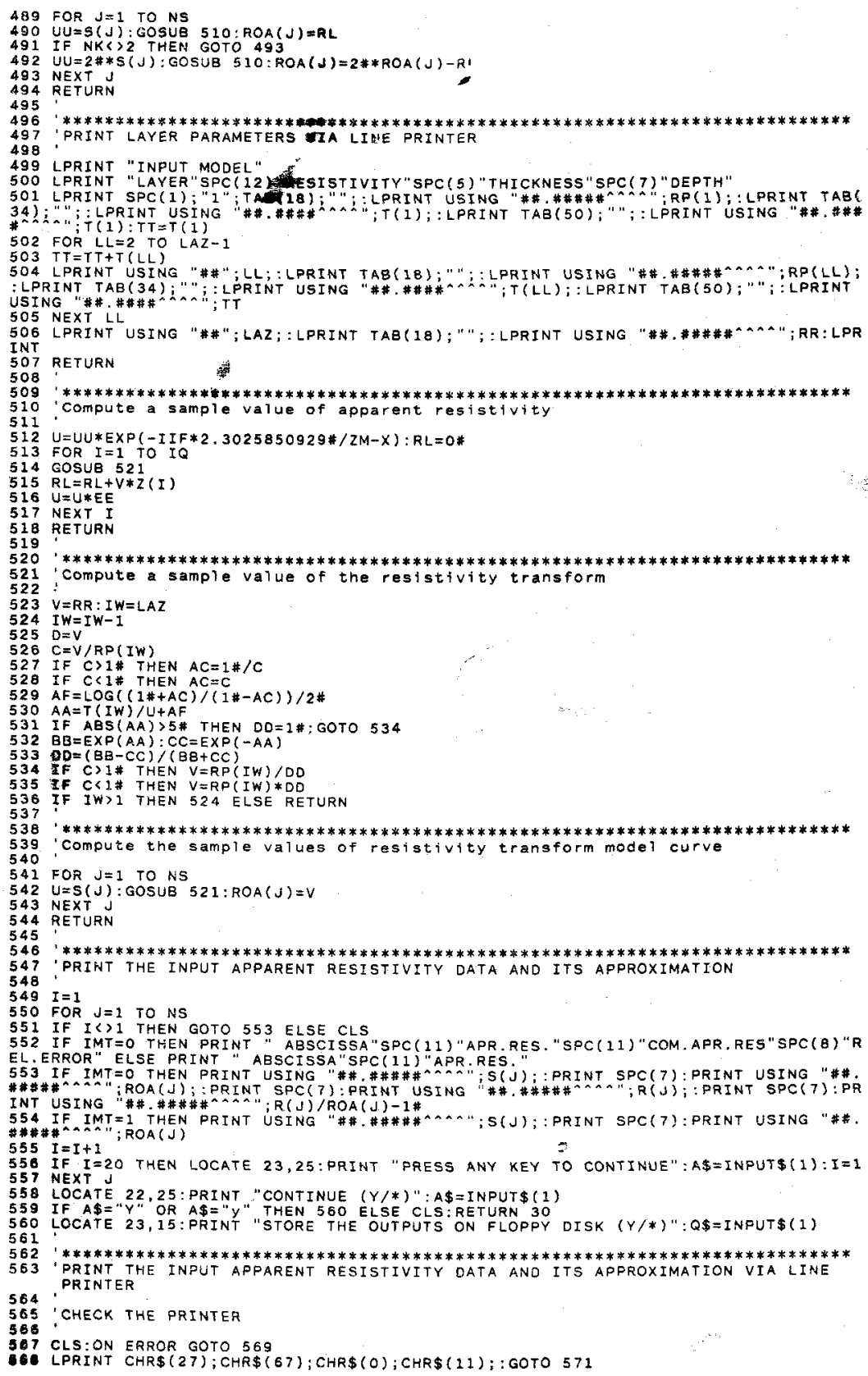




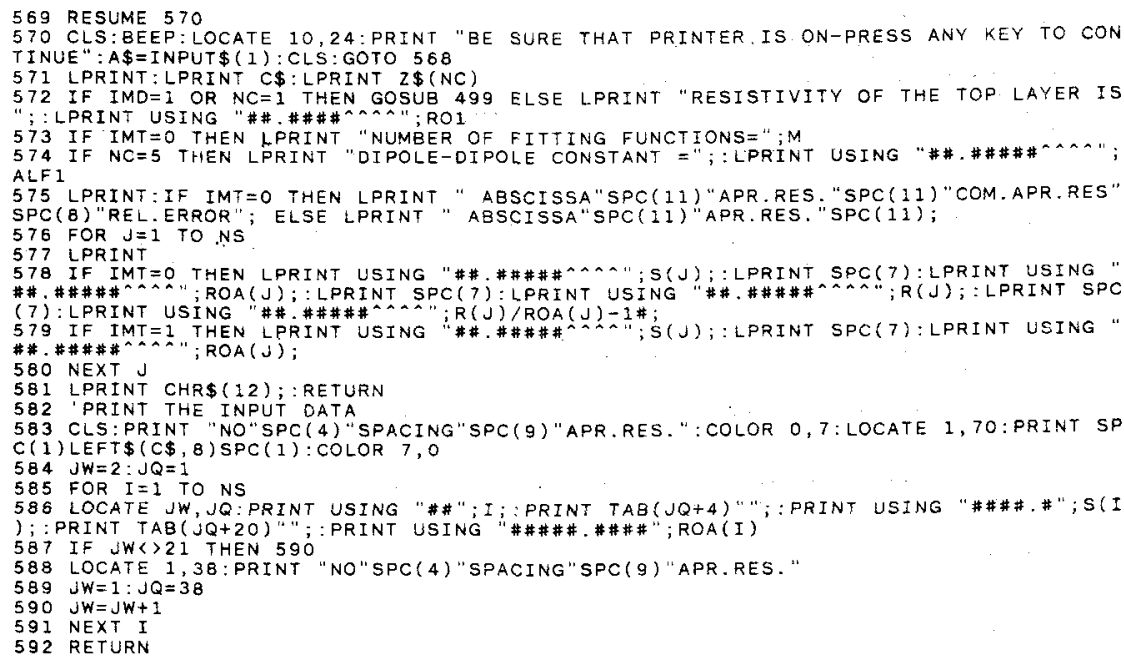

results has not been published till now. For this reason the two-electrode filter has been used and a sample value of Wenner apparent resistivity model curve was obtained from two sample values of two-electrode apparent resistivity (Das and Verma 1980).

\section{Conclusion}

A computer program listed in the Appendix has been presented to carry out three types of transformation of VES data by the least-squares method and to compute VES model curves by the linear filter method. The program has been developed for four electrode configurations (twoelectrode, Wenner, Schlumberger and dipole-dipole configurations). However, any other array may be included into the program. Field data and outputs can be stored on disk for further processes, e.g. plotting the curves, direct or iterative interpretations and so on.

The program can be run on IBM-PC compatible microcomputers and permits in-field processing of VES data demonstrated by an example.

\section{Acknowlegements}

I am grateful to Dr. Y. Murakami for providing his filter coefficients and for helpful comments, and to Dr. J. Vandenberghe for critically reading the manuscript. 


\section{REFERENCES}

BAŞOKUR, A.T. 1984a. A numerical direct interpretation method of resistivity soundings using the Pekeris model. Geophysical Prospecting 32, 1131-1146.

BAŞOKUR, A.T. 1984b. The use of two-electrode and Schlumberger filters for computing resistivity and EM sounding curvers,. Geophysical Prospecting 32, 132-138.

DAS, U. C. and VFRMA, S.K. 1980. Digital linear filter for computing type curves for the twoelectrode system of resistivity sounding. Geophysical Prospecting 28, 610-619.

GHOSH, D.P. 1971. The application of linear filter theory to the direct interpretation of geoelecrical resistivity sounding measurements. Geophysical Prospecting 19, 192-127.

KOEFOED, 0. 1970. A fast method for determining the layer distribution from the raised kernel function in geoelectrical soundings. Geophysical Prospecting 18, 564-570.

KOEFOED, O. 1979. Gosoundings Principles 1, Resistivity sounding measurements. Elsevier, Amsterdam.

KOHLBECK, F. 1985. Computing the kernel function in resistivity sounding with an arbitrary electrode configuration. Geophysical Prospecting 33, 128-137.

KUMAR, R. and CHOWDARY, M.V.R. 1982. A numerical method to compute the resistivity transform from Wenner sounding data. Geophysical Prospecting 30, 898-909.

KUNETZ, G. 1966. Principles of direct current resistivity prospecting. Gebriider Borntraeger, Berlin.

MURAKAMI, Y., ZFRILLI, A. and BISDORF R. J. 1984. Improved digital filters for the calculation of Schlumberger sounding curves by convointion, USGS Open-File Report 84-280.

O'NEILL, N. P. and MERRICK, N.: P. 1984. A digital linear filter for resistivity sonnding with a generalized electrode array. Geophysical Prospecting 32, 105-123.

PEKERIS, C.I. 1940. Direct method of interpretation in resistivity prospecting. Geophysics 5 , $31-46$.

SANTINI, R. and ZAMBRANO, R. 1981. A numerical method of calculating the kernel function from Schlumberger apparent resistivity data. Geophysical Prospecting, 29, 108-127.

SANTINI, R. and ZAMBRANO, R. 1982. Reply to comment by E. Szaranies. Geophysical Prospecting 30, 949.

SANTINI, R. and ZAMBRANO, R. 1983. A generalized method to calculate standart curves for geoeleetrical soundings. Genexploration 21, 91-103. 